\title{
Relationship between sensory processing sensitivity and hypochondriacal features and the moderating role of somatic symptoms
}

\author{
Siemen Dal
}

Maastricht University

s.dal@student.maastrichtuniversity.nl

\begin{abstract}
Background. Only very little is known about the etiology of hypochondriasis, a mental disorder characterized by the preoccupied beliefs or fears of having a serious disease or being in a life-threatening condition and the elevated focus on own physical symptoms. A relatively new concept named sensory processing sensitivity (SPS) is about the sensitivity towards stimuli and is related to an elevated self-focus. Due to its relation with an elevated self-focus, SPS might be related to hypochondriacal features. The present study investigated this relationship. Moreover, in the light of recent reformulation of hypochondriasis in the DSM-5 in illness anxiety disorder and somatic symptom disorder, this study also examined how the presence of somatic complaints affects the relationship between SPS and hypochondriacal features. Methods. A cross-sectional questionnaire study was conducted in which 302 participants from the general population were enrolled. These participants were recruited through several ways online and at several physical locations. Hypochondriacal fears and beliefs were assessed using the Illness Attitudes Scale (IAS), while SPS was assessed using the Highly Sensitive Person scale (HSP). At last, having physical complaints was assessed with descriptive items on having complaints and their duration and location. Results. The present study found that HSP and its subscales Ease of Excitation and Low Sensory Threshold were positively and significantly correlated to IAS and its subscales Health Anxiety and Illness Behavior. In contrast, it was found that the Aesthetic Sensitivity subscale of the HSP scale was not correlated to IAS or its subscales. Furthermore, a moderation test using linear regression analyses showed that having somatic complaints did not moderate the relationship between HSP and IAS. Having specifically chronic somatic complaints, however, did tend to moderate this relationship. Conclusion. The present study is the first study to show the importance of a high SPS
\end{abstract}


in having hypochondriacal fears and beliefs and the role of somatic complaints in this relationship. However, future research is needed to further understand this relationship and to investigate its causality.

\section{Keywords}

Hypochondriasis, sensory processing sensitivity, somatic complaints, Highly Sensitive Person scale, Illness Attitudes Scale.

\section{Introduction}

Hypochondriasis is a chronic disorder characterized by the preoccupied belief or anxiety of having a serious disease or being in a life-threatening condition, causing distress or impairment in social, occupational or other important areas of functioning. These beliefs or fears are based on one's misinterpretation of its physical symptoms. Consequently, these physical symptoms or sensations experienced as abnormal elicit and confirm their hypochondriacal beliefs and fears. Despite the fact that physicians reassure hypochondriasis patients of their health, the excessive worrying persists (American Psychiatric Association, 2000). Hypochondriasis, however, is a diagnosis in the DSM IV, while the most recent version of the handbook is DSM-5. With the recent introduction of DSM-5, changes have been made to the classification of somatoform disorders and hypochondriasis is now distinguished in somatic symptom disorder and illness anxiety disorder - health anxiety with somatic complaints and health anxiety without somatic complaints (Mayo Clinic). Nevertheless, the term hypochondriasis is still used in the clinical field and in the research field to refer to this disease.

While much is known about the clinical features of hypochondriasis, knowledge on the etiology of hypochondriasis is limited, with only adverse childhood experiences of illness being suggested as contributor to the development of hypochondriacal traits in later life (Barsky, Wool, Barnett, \& Cleary, 1994; Bianchi, 1971; Mabe, Hobson, Jones, \& Jarvis, 1988; Noyes et al., 2002). Sensory processing sensitivity (SPS) is a relatively new concept which might be proposed to be related to hypochondriasis. SPS is an innate trait coined by Aron (Aron \& Aron, 1997), which is best explained with its extremes. Highly sensitive people are characterized by higher sensitivity to noises, bright lights and strong smells. Moreover, these people have a rich and complex inner life, get frightened easily and get rattled when having to accomplish a lot in a short time. In other words: highly sensitive people are more introvert, sensitive to stimuli and vigilant, where lower SPS is related to being extravert, less sensitive to stimuli and explorative. Additionally, those who are highly 
sensitive are more aware of subtleties and process information on a deeper and more reflective way than those with lower SPS (Aron \& Aron, 1997). Thus, it seems reasonable that a high SPS, characterized by being more reflective and self-focused, might be related to hypochondriasis, characterized by an elevated focus on bodily sensations.

Due to its novelty, research on SPS is limited. It has been suggested that SPS is associated with social anxiety (Neal, Edelmann, \& Glachan, 2002), avoidant personality (Meyer \& Carver, 2000), agoraphobic avoidance (Hofmann \& Bitran, 2007), anxiety and depression (Liss, Timmel, Baxley, \& Killingsworth, 2005) and stress and ill-health (Benham, 2006). A more elaborated study focused on specific components of SPS, namely ease of excitation, low sensory threshold and aesthetic sensitivity, and found that its different components were related to different disorders such as autism, anxiety and depression (Liss, Mailloux, \& Erchull, 2008). Research on the relationship between SPS and hypochondriacal fears and beliefs, however, is missing. Therefore, the present study will investigate exactly this relationship.

Besides examining the plain relationship between SPS and hypochondriacal traits, this study will also look further into this relationship by studying it in two groups of participants: those who suffer from somatic symptoms and those who do not. This distinction is made interesting as a result of the redefinition of hypochondriasis by distinguishing it in somatic symptom disorder and illness anxiety disorder on the basis of having somatic complaints or not. Literature on the role of somatic symptoms in health anxiety, however, is missing. One study found that somatization and pain-related dysfunction are positively correlated (Riley III \& Robinson, 1998), where another study found a positive correlation between SPS and somatic symptoms (Benham, 2006). Therefore, one might predict that SPS, somatic symptoms and hypochondriacal fears and beliefs are all positively related to each other. Moreover, it seems reasonable that having somatic symptoms moderates the relationship between a high SPS - characterized by an elevated self-focus - and hypochondriacal fears and beliefs - characterized by an elevated focus on own physical sensations.

In conclusion, the first research question is as follows: is a high SPS a predictor for hypochondriacal fears and beliefs? Moreover, to understand the role of somatic symptoms in this relationship, the second research question is: is the relationship between SPS and hypochondriacal fears and beliefs stronger in presence of somatic complaints than in absence of somatic complaints? In line with past studies, the first hypothesis is that a high SPS is a significant predictor of hypochondriacal fears and beliefs. Similarly in line with 
past studies, the second hypothesis is that SPS is a better predictor of hypochondriacal features in presence of somatic symptoms than in absence of somatic symptoms.

\section{Material and methods}

\section{Participants}

The study sample population included 302 participants. Within these 302 participants, $237(78.5 \%)$ were women and 65 (21.5\%) were men. Moreover, 147 (48.7\%) participants reported to have physical complaints and 155 (51.3\%) participants reported not to have any physical complaints. To be able to participate in this study, participants had to be aged between 18 and 65 years old. Similarly, being mentally competent was also an inclusion criterion for participation. The main exclusion criterion for this study was being pregnant. These participants were recruited from the general population through four different ways online and at ten different physical locations in four different cities or villages using posters. By participating, participants had the opportunity to join a raffle to win prizes.

\section{Measures and design}

To assess all relevant constructs, the present study used a cross-sectional questionnaire design. The used questionnaire consisted of, among others, The Illness Attitudes Scale (IAS), the Highly Sensitive Person scale (HSP) and descriptive items on having complaints and the location and duration of these complaints. The IAS is a 28 -item questionnaire assesssing attitudes and concerns about illness and health and is comprised of two subscales, namely Health Anxiety and Illness Behavior (Speckens \& Van Hemert). This questionnaire is suggested to be the gold standard for assessing hypochondriacal fears and beliefs, due to its clinimetric properties and high sensitivity (Sirri, Grandi, \& Fava, 2007). Furthermore, the 27-item questionnaire HSP was used to assess SPS. The HSP is comprised of three subscales: Ease of Excitation (EOE), Aesthetic Sensitivity (AES) and Low Sensory Threshold (LST). EOE assesses the experiences of being mentally overwhelmed by external or internal stimuli, AES assesses aesthetic awareness and LST assesses unpleasant sensory arousal (Smolewska, McCabe, \& Woody, 2006).

\section{Results}

Using Pearson's correlation test, bivariate correlations were calculated between the HSP scale and the EOE, AES and LST subscales on the one hand and the IAS scale and the Health Anxiety and IIIness Behavior subscales on the other hand. Responses on the HSP scale were positively and significantly correlated with the responses on the IAS scale $(r=.43, p=.00)$ and the responses on the Health Anxiety $(r=.34, p=.00)$ and IIIness 
than model $2, \mathrm{R}^{2}$ change $=.00, p=.34$. Additionally, the interaction term in model 3 has no significant effect on hypochondriacal fears and beliefs, $b=.08, t(298)=.96, p=.34$. These findings suggest that there is no potential moderation between somatic symptoms and SPS on hypochondriacal traits.

Next, a similar hierarchical multiple regression was conducted with selected data of only the participants with somatic complaints for longer than 1 year $(N=85)$ and the participants without any somatic complaints $(N=155)$. Similarly, model 3 with the added interaction term does again not account for significantly more variance than model $2, R^{2}$ change $=.01, p=.09$. Moreover, the interaction term in model 3 has again no significant effect on hypochondriacal fears and beliefs, $b=.16, t(298)=1.73, p=.09$. However, when comparing the results of this moderation analyses with specifically chronic somatic complaints with the previous moderation analyses with general somatic complaints, it is noteworthy that the p-value has decreased from .34 towards .09, reaching a significance level below 0.1. Although the moderating role of chronic somatic complaints is still not significant, this trend is at least interesting. Plots of the relationship between HSP and IAS with somatic complaints and chronic somatic complaints as subgroups are presented in Figure 1. Figure $1 b$ displays that the relationship between HSP and IAS was stronger in presence of chronic somatic symptoms than without chronic somatic symptoms and thereby displays the trend of chronic somatic symptoms having a positively moderating role. Moreover, it confirms that chronic somatic complaints (b) tend to moderate the relationship between HSP and IAS more than general somatic complaints (a), which was shown statistically.
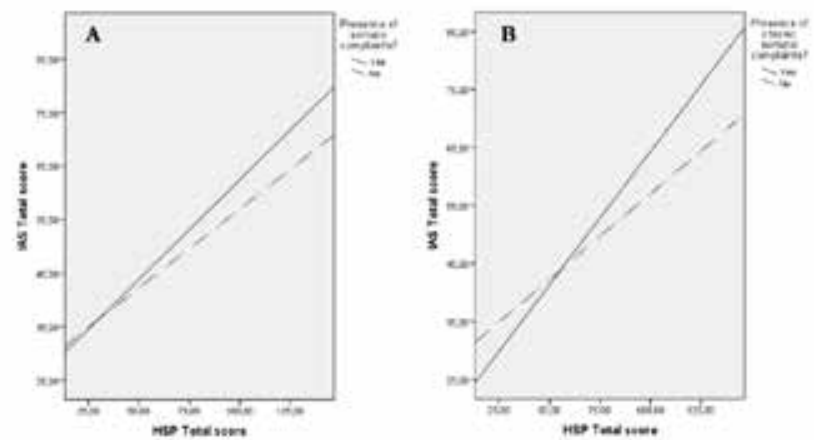

Figure 1. A) Plot of the interaction between HSP and somatic complaints in predicting IAS. B) Plot of the interaction between HSP and chronic somatic complaints in predicting IAS. 


\section{Discussion/Conclusion}

The goal of the present study was to broaden the understanding of the etiology of hypochondriacal features, a huge gap in the literature. Therefore, the present study aimed to investigate the relationship between SPS and hypochondriacal fears and beliefs and the potential moderating role of somatic symptoms in this relationship.

The first hypothesis was that a high SPS would predict hypochondriacal fears and beliefs, based on the reasoning that being more self-focused - a characteristic of a high SPS (Aron \& Aron, 1997) - seems to be related to hypochondriacal features, where attention is focused on own bodily sensations (American Psychiatric Association, 2000). Results of this study indicate that the HSP scale and the subscales EOE and LST were correlated to IAS and its subscales Health Anxiety and Illness Behavior on a moderately positive way. In contrast, the AES subscale was not significantly correlated to IAS or its subscales at all. Thus, this study suggests that a higher SPS, or at least a higher EOE or LST, is related to more hypochondriacal fears and beliefs in general, or at least more health anxiety or illness behavior. Therefore, the first hypothesis that a high SPS would predict hypochondriacal fears and beliefs is fully supported.

The second hypothesis was that the relationship between a high SPS and hypochondriacal fears and beliefs would be stronger in presence of somatic symptoms than in absence of somatic symptoms, as it seemed reasonable that having somatic symptoms moderates the relationship between an elevated self-focus - a characteristic of high SPS - and an elevated focus on own physical symptoms - a hypochondriacal feature. Results of the present study indicate that the presence of somatic symptoms is not a significant moderator in the relationship between SPS and hypochondriacal fears and beliefs. Therefore, the second expectation cannot be supported. However, when only taking participants with chronic physical complaints - physical complaints for longer than 1 year - into account, the present study found that the presence of chronic somatic symptoms in particular did tend to positively moderate the relationship between SPS and hypochondriacal fears and beliefs. This finding implies that having chronic somatic complaints increases the probability of having hypochondriacal fears and beliefs when one has a high SPS.

In conclusion, the present study is the first study to show the importance of a high SPS in having hypochondriacal fears and beliefs by having found the moderately positive relationship. Additionally, the present study is also the first study to investigate the role of somatic complaints in this relationship having found that somatic complaints did not 
moderate this relationship, but that specifically chronic somatic complaints did tend to moderate it. Major strengths of the present study are its diverse study sample of 302 participants, the multiple methods of recruiting participants and the use of validated measurement instruments. Despite these strengths, the present study also has several limitations. Limitations of the present study are its cross-sectional design, which does not allow to make conclusions on causality, and the non-normal distribution of important study sample characteristics such as age and sex. Various suggestions for future research arise from these limitations, such as examining the causal relationship between having a high SPS and developing hypochondriacal fears and beliefs using a longitudinal study design. Thus, the present cross-sectional questionnaire study shed light on a relationship that was yet to be studied and thereby opened doors for future research.

\section{Role of the student}

Siemen Dal was an undergraduate student working under the supervision of Linda Vancleef when the research in this report was performed. The collection of data was mainly done by the supervisor. The proposal of the topic, the processing of the results as well formulation of the conclusions and the writing were done by the student.

\section{References}

1. American Psychiatric Association. (2000). Diagnostic and statistical manual of mental disorders, text revision (DSM-IV-TR): American Psychiatric Association.

2. Aron, E. N., \& Aron, A. (1997). Sensory-processing sensitivity and its relation to introversion and emotionality. Journal of personality and social psychology, 73(2), 345

3. Barsky, A. J., Wool, C., Barnett, M. C., \& Cleary, P. D. (1994). Histories of childhood trauma in adult hypochondriacal patients. The American journal of psychiatry.

4. Benham, G. (2006). The highly sensitive person: Stress and physical symptom reports. Personality and individual differences, 40(7), 1433-1440.

5. Bianchi, G. (1971). Origins of disease phobia. Australian and New Zealand Journal of Psychiatry, 5(4), 241-257.

6. Hofmann, S. G., \& Bitran, S. (2007). Sensory-processing sensitivity in social anxiety disorder: relationship to harm avoidance and diagnostic subtypes. Journal of anxiety disorders, 21(7), 944-954.

7. Liss, M., Mailloux, J., \& Erchull, M. J. (2008). The relationships between sensory processing sensitivity, alexithymia, autism, depression, and anxiety. Personality and individual differences, 45(3), 255-259.

8. Liss, M., Timmel, L., Baxley, K., \& Killingsworth, P. (2005). Sensory processing sensitivity and its relation to parental bonding, anxiety, and depression. Personality and individual differences, 39(8), 1429-1439.

9. Mabe, P. A., Hobson, D. P., Jones, L. R., \& Jarvis, R. G. (1988). Hypochondriacal traits in medical inpatients. General Hospital Psychiatry, 10(4), 236-244. 
10. Mayo Clinic. DSM-5 redefines hypochondriasis. http://www.mayoclinic.org/medical-professionals/ clinical-updates/psychiatry-psychology/diagnostic-statistical-manual-mental-disorders-redefineshypochondriasis

11. Meyer, B., \& Carver, C. S. (2000). Negative childhood accounts, sensitivity, and pessimism: A study of avoidant personality disorder features in college students. Journal of Personality Disorders, 14(3), 233-248.

12. Neal, J. A., Edelmann, R. J., \& Glachan, M. (2002). Behavioural inhibition and symptoms of anxiety and depression: Is there a specific relationship with social phobia? British Journal of Clinical Psychology, 41(4), 361-374.

13. Noyes, R., Stuart, S., Langbehn, D. R., Happel, R. L., Longley, S. L., \& Yagla, S. J. (2002). Childhood antecedents of hypochondriasis. Psychosomatics, 43(4), 282-289.

14. Riley III, J. L., \& Robinson, M. E. (1998). Validity of MMPI-2 profiles in chronic back pain patients: Differences in path models of coping and somatization. The Clinical journal of pain, 14(4), 324-335.

15. Sirri, L., Grandi, S., \& Fava, G. A. (2007). The Illness Attitude Scales. A clinimetric index for assessing hypochondriacal fears and beliefs. Psychotherapy and Psychosomatics, 77(6), 337-350.

16. K. A., McCabe, S. B., \& Woody, E. Z. (2006). A psychometric evaluation of the Highly Sensitive Person Scale: The components of sensory-processing sensitivity and their relation to the BIS/BAS and "Big Five". Personality and individual differences, 4O(6), 1269-1279.

17. Speckens, A. E. M., \& Van Hemert, A. M. MENINGEN OVER ZIEKTE EN GEZONDHEID. from http://www. psychiatrieweb.mywebhome.nl/pw.somatisatie/files/docs/ias.pdf 